EPJ Web of Conferences 41, 03018 (2013)

DOI: $10.1051 /$ epjconf/20134103018

(C) Owned by the authors, published by EDP Sciences, 2013

\title{
Ultrafast carrier dynamics and radiative recombination in multiferroic $\mathrm{BiFeO}_{3}$ single crystals and thin films
}

\author{
Y. M. Sheu ${ }^{1}$, S. A. Trugman ${ }^{1}$, J. Xiong ${ }^{1}$, Y.-S. Park ${ }^{1}$, S. Lee ${ }^{2}$, H. T. Yi ${ }^{3}$, S.-W. Cheong ${ }^{3}$, Q. X. Jia ${ }^{1}$, \\ A. J. Taylor ${ }^{1}$, and R. P. Prasankumar ${ }^{1}$ \\ ${ }^{1}$ Center for integrated Nanotechnologies, MS K771, Los Alamos National Laboratory, Los Alamos, \\ New Mexico 87545, USA \\ ${ }^{2}$ Korea Atomic Energy Research Institute, Daejeon 305-353, South Korea \\ ${ }^{3}$ Rutgers Center for Emergent Materials and Department of Physics and Astronomy, Rutgers \\ University, 136 Frelinghuysen Rd., Piscataway, New Jersey 08854, USA
}

\begin{abstract}
We report a detailed comparison of ultrafast carrier dynamics in single crystals and thin films of multiferroic $\mathrm{BiFeO}_{3}$ (BFO). Using degenerate femtosecond optical pump-probe spectroscopy, we find that the observed dynamics are qualitatively similar in both samples. After photoexcitation, electrons relax to the conduction band minimum through electron-phonon coupling, with subsequent carrier relaxation proceeding via various recombination pathways that extend to a nanosecond timescale. Subtle differences observed in our measurements indicate that BFO films have a higher band gap than single crystals. Overall, our results demonstrate that carrier relaxation in $\mathrm{BFO}$ is analogous to that in bulk semiconductors.
\end{abstract}

\section{Introduction}

Bismuth ferrite (BFO) is one of the most actively studied multiferroic materials due to its room temperature coexistence of ferroelectricity (FE) $\left(\mathrm{T}_{\mathrm{c}} \sim 1100 \mathrm{~K}\right)$ and antiferromagnetism (AFM) $\left(\mathrm{T}_{\mathrm{N}} \sim 640 \mathrm{~K}\right)$. Much research has focused on enhancing their weak mutual coupling, particularly by using growth techniques that vary the structure or strain in BFO films [1-3]. This could allow control of magnetism with electric fields or vice versa, which would lead to a variety of potential applications.

Despite the intense research on this material, relatively few studies of its ultrafast dynamics have been done to date [4-7]. Previously, we have studied carrier relaxation and recombination in BFO single crystals up to $800 \mathrm{~K}$ by using ultrafast optical (UOS) and photoluminescence (PL) spectroscopies [5]. Here, we extend these studies to compare carrier dynamics in bulk and thin film $\mathrm{BFO}$, revealing that the dynamics are quite similar throughout the measured temperature range, with subtle differences that can be linked to the higher band gap of BFO films.

\section{Experimental setup and samples}

We use degenerate ultraviolet (UV) pump-probe spectroscopy with photon energies $\left(E_{\mathrm{ph}}\right)$ tunable from 3.12-3.23 eV, generated from second harmonic generation in BBO crystals. The details of the

This is an Open Access article distributed under the terms of the Creative Commons Attribution License 2.0, which permits unrestricted use, distribution, and reproduction in any medium, provided the original work is properly cited. 
pump-probe and PL setups can be found in ref. [5]. A BFO (001) pc bulk single crystal (SC) with 220 $\mu \mathrm{m}$ thickness was grown by $\mathrm{Bi}_{2} \mathrm{O}_{3}$ flux. The $50 \mathrm{~nm}$ BFO thin film (TF) was deposited on a $\mathrm{SrTiO}_{3}$ (001) substrate through laser molecular beam epitaxy, using a $\mathrm{XeCl}$ excimer laser with a wavelength of $308 \mathrm{~nm}$ and an energy density of $3 \mathrm{~J} / \mathrm{cm}^{2}$ on the target surface.

\section{Experimental results and discussion}
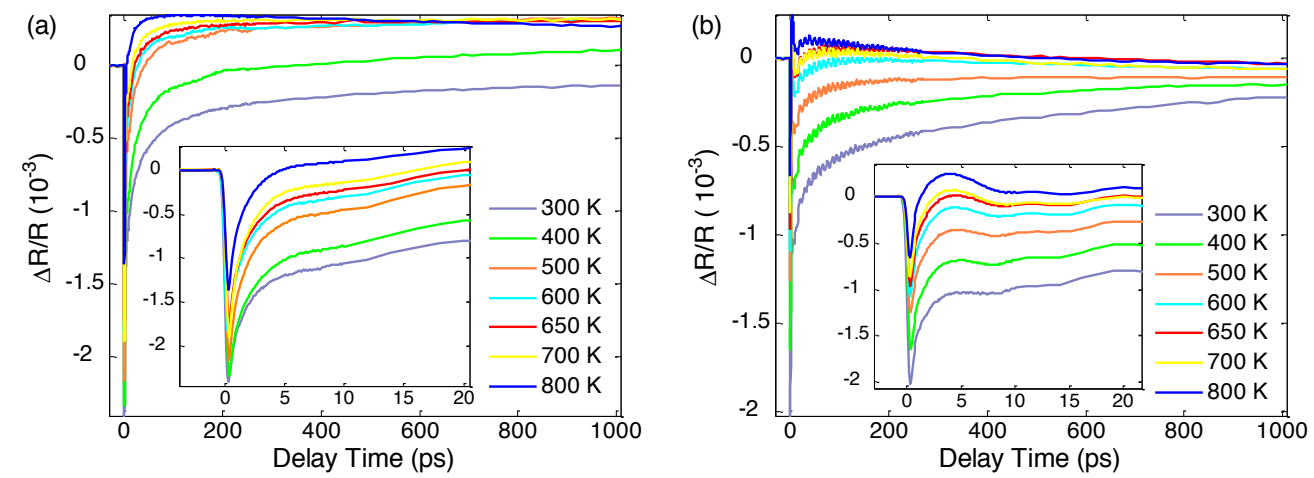

Fig. 1. Degenerate temperature-dependent pump-probe experiments at $3.14 \mathrm{eV}$ on the (a) $\mathrm{SC}$ and (b) TF samples. The insets display the early time dynamics.

Figures 1(a) and 1(b) display the temperature dependence of the carrier dynamics from the SC and TF, respectively, following $3.14 \mathrm{eV}$ excitation (above the $E_{g} \sim 2.7 \mathrm{eV}$ band gap) with an absorbed pump fluence of $\sim 36 \mu \mathrm{J} / \mathrm{cm}^{2}$. The observed dynamics are qualitatively similar in both samples. Immediately following photoexcitation, the normalized photoinduced change in reflectivity $(\Delta \mathrm{R} / \mathrm{R}<0)$ drops due to the photoinduced increase in carrier density $(\Delta \mathrm{N})$ that bleaches the absorption through state filling. The subsequent positive reflectivity change $(\Delta \mathrm{R} / \mathrm{R}>0)$ results from an increase in lattice temperature $\left(\Delta \mathrm{T}_{\mathrm{L}}\right)$ given by the excess photon energy $(\Delta E)$ above $E_{g}$, as the photoexcited electrons lose their energy to phonons while relaxing to the conduction band minimum [5]. The overall sign of $\Delta \mathrm{R} / \mathrm{R}$ at early times is thus determined by the competition between $\Delta \mathrm{N}$ and $\Delta \mathrm{T}_{\mathrm{L}}$ [5].

Each trace is best fitted by three time constants with magnitudes of $\sim 1$ picosecond (ps) (fast), $\sim 10-50$ ps (intermediate), and $\sim 1-3$ nanoeconds (ns) (slow) at all measured temperatures. The fast time constant is due to electron-phonon coupling, as discussed above, and has no significant temperature dependence up to $800 \mathrm{~K}$ in both samples [5]. The intermediate time constant, previously attributed to radiative recombination, and the slow time constant, attributed to both heat diffusion and recombination [5], are also similar in both samples from 300-800 K. The primary differences in carrier dynamics between the two samples are the temperature and time delay at which the zero crossings in the $\Delta \mathrm{R} / \mathrm{R}$ signals (ZC) (insets of Fig. 1) and the oscillations observed in $\Delta R / R$ for the film. The latter is due to a propagating strain wave (acoustic phonon) created when the photoexcited carriers transfer their energy to the lattice, as observed in many other studies of similar materials [7].

More insight into the difference between the $\mathrm{ZC}$ for the bulk and film samples can be obtained by considering that the dynamics in the film have the same dependence on $E_{p h}$ at room temperature as that in the bulk (not shown here) [5]. However, the $E_{\mathrm{ph}}$ required to observe the ZC in the film is higher than in the bulk. Therefore, when we slightly increase $E_{\mathrm{ph}}$ to $3.22 \mathrm{eV}$, the overall $\Delta \mathrm{R} / \mathrm{R}$ signal after $\sim 1$ ps shifts and crosses zero at a lower temperature $\left(T_{\mathrm{R} 0} \sim 400 \mathrm{~K}\right.$ vs. $\sim 700 \mathrm{~K}$ for $\left.3.14 \mathrm{eV}\right)$ (Figure 2(a)). This is an indication that $T_{R 0}$ is insensitive to the AFM transition at $T_{N}$ and the sign of $\Delta \mathrm{R} / \mathrm{R}$ strongly depends on the magnitude of $\Delta E[5]$.

Since $E_{\mathrm{ph}}$ of the pump is close to $E_{g}$ and the reflectivity above $E_{g}$ is very sensitive to $\Delta \mathrm{T}_{\mathrm{L}}$ [5], any changes in the band structure would change $\Delta E$ and in turn the sign of $\Delta \mathrm{R} / \mathrm{R}$, due to the competition between $\Delta \mathrm{N}$ and $\Delta \mathrm{T}_{\mathrm{L}}$. Figure 2(b) shows time-integrated PL measurements on both samples. While direct band-to-band recombination is observed in the SC (sharp peak in Figure 2(b)), it disappears in 
the TF, which emits a much broader PL spectrum that is centered at lower energies. This is likely due to defect state emission, although the nature of the broad emission in both samples is not definitively known [5].
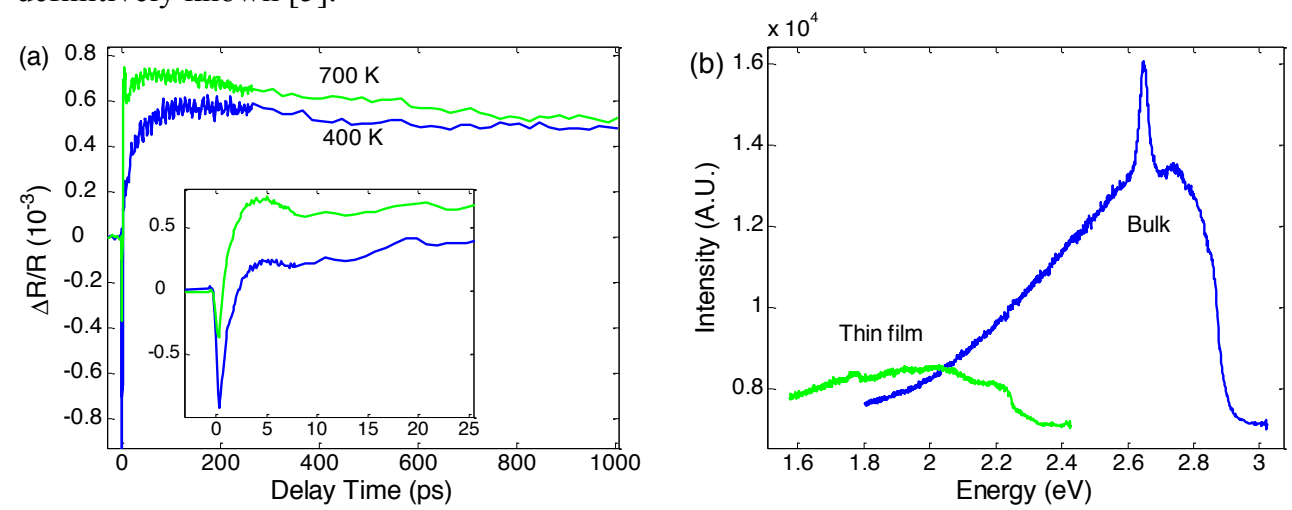

Fig. 2. (a) Degenerate $3.22 \mathrm{eV}$ pump-probe measurements on the TF, with an inset displaying the early time dynamics. (b) PL spectrum measured after $3.06 \mathrm{eV}$ excitation at room temperature in both samples.

The fact that $E_{\mathrm{ph}}$ required to observe the $\mathrm{ZC}$ is higher in the film than in the bulk suggests that the TF has a higher band gap than the SC. This can explain the disappearance of the sharp peak in the PL spectrum for the film, since the absorption at a given $E_{\mathrm{ph}}$ is lower in the film and the excitation volume is also smaller than in the bulk. This hypothesis of a higher band gap is supported by previous work on different film structures [6].

Finally, it is worth emphasizing that the intermediate time constant is not due to spin-lattice relaxation [5], as Figures 1(b) and 2(a) clearly show a monotonic decrease in this time constant across $\mathrm{T}_{\mathrm{N}}$. As previously mentioned, we attribute this time constant to radiative recombination, but its detailed nature is still unclear due to the different possible recombination pathways in both samples.

In conclusion, we have studied ultrafast carrier dynamics in BFO single crystals and thin films. The observed dynamics are qualitatively similar for both samples, with the primary difference (the ZC) mainly originating from differences in their electronic structure. Our fluence $(\sim 0.024-0.5$ $\mathrm{mJ} / \mathrm{cm}^{2}$ ), energy (including $\sim 3.15 \mathrm{eV}$ pump and $1.55 \mathrm{eV}$ probe) and temperature $(10 \mathrm{~K}-800 \mathrm{~K})$ dependent measurements (not all shown here) consistently support this, revealing a $\sim 1$ ps electronphonon coupling time and recombination on nanosecond time scales, with no evidence of a spinlattice contribution. Overall, our results suggest that carrier relaxation in BFO, despite the presence of FE and AFM order, is largely analogous to that in a bulk semiconductor.

\section{References}

1. J. Wang, J. B. Neaton, H. Zheng, V. Nagarajan, S. B. Ogale, B. Liu, D. Viehland, V. Vaithyanathan, D. G. Schlom, U. V. Waghmare, et al., Science 299, 1719 (2003)

2. B. Dupé, I. C. Infante, G. Geneste, P.-E. Janolin, M. Bibes, A. Barthélémy, S. Lisenkov, L. Bellaiche, S. Ravy, and B. Dkhil, Phys. Rev. B 81, 144128 (2010)

3. W. Ratcliff, D. Kan, W. Chen, S. Watson, S. Chi, R. Erwin, G. J. McIntyre, S. C. Capelli, and I. Takeuchi, Adv. Funct. Mater. 21, 1567 (2011)

4. K. Takahashi, N. Kida, and M. Tonouchi, Phys. Rev. Lett. 96, 117402 (2006)

5. Y. M. Sheu, S. A. Trugman, Y.-S. Park, S. Lee, H. T. Yi, S.-W. Cheong, Q. X. Jia, A. J. Taylor, and R. P. Prasankumar, Appl. Phys. Lett. 100, 242904 (2012)

6. Zuanming Jin, Yue Xu, Zhengbing Zhang, Gaofang Li, Xian Lin Guohong Ma, Zhenxiang Cheng, and Xiaolin Wang, Appl. Phys. Lett. 100, 071105 (2012)

7. P. Ruello, T. Pezeril, S. Avanesyan, G. Vaudel, V. Gusev et al., Appl. Phys. Lett. 100, 212906 (2012) 